\title{
Acoustic positioning system for KM3NeT
}

\section{S. Viola}

INFN Laboratori Nazionali del Sud, Via S.Sofia 62, 95123, Catania, Italy

E-mail: sviola@lns.infn.it

\section{Ardid}

Instituto de Investigación para la Gestión Integrada de las Zonas Costeras, Universitat Politècnica de València, Gandia, Spain

E-mail: mardidefis.upv.es

\section{Bertin}

Centre de Physique des Particules de Marseille, Marseille, France

E-mail: bertin@cppm.in2p3.fr

\section{R. Lahmann}

Friedrich-Alexander-Universität Erlangen-Nürnberg, Centre for Astroparticle Physics,

Erwin-Rommel-Str. 1, 91058 Erlangen, Germany

E-mail: robert.lahmann@physik.uni-erlangen.de

\section{Pellegrino}

INFN Sezione Bologna, V.le Berti Pichat 6/2, 40127, Bologna, Italy

Dipartimento di Fisica Università di Bologna, V.le Berti Pichat 6/2, 40127, Bologna, Italy

E-mail: carmelo.pellegrino@bo.infn.it

\section{G. Riccobene}

INFN Laboratori Nazionali del Sud, Via S.Sofia 62, 95123, Catania, Italy

E-mail: riccobenedlns.infn.it

\section{Saldaña}

Instituto de Investigación para la Gestión Integrada de las Zonas Costeras, Universitat Politècnica de València, Gandia, Spain

E-mail: masalcos@upv.es

\section{P. Sapienza*}

INFN Laboratori Nazionali del Sud, Via S.Sofia 62, 95123, Catania, Italy

E-mail: sapienza@lns.infn.it

\section{F. Simeone}

INFN Sezione Roma, P.le A. Moro 2, 00185, Roma, Italy

E-mail: francesco.simeonedromal.infn.it

\section{for the KM3NeT Collaboration}


$\mathrm{KM} 3 \mathrm{NeT}$ is the next generation neutrino telescope in the Mediterranean Sea employing the technique of Cherenkov photon detection. The Acoustic Positioning System (APS) is a mandatory sub-system of KM3NeT that must provide the position of the telescope's mechanical structures, in a geo-referenced coordinate system. The APS is important for a safe and accurate deployment of the mechanical structures and, for the sake of science, for precise reconstruction of neutrinoinduced events. The KM3NeT APS is composed of three main sub-systems: 1) an array of acoustic receivers rigidly connected to the telescope mechanical structures; 2) a Long Base-Line (LBL) of acoustic transmitters (beacons) and receivers, anchored on the seabed at known positions; 3 ) a farm of PCs for the acoustic data analysis, on-shore. On shore, the positions of the acoustic receivers are calculated by measuring the ToF (Time Of Flight) of the LBL beacons' signals on the acoustic receivers, thus determining, via multi-lateration, the position of the acoustic receivers with respect to the geo-referenced LBL. The synchronized and syntonized electronics and the data transmission/acquisition allows for calculating the latencies of the whole data acquisition chain with an accuracy of better than $100 \mathrm{~ns}$. The APS, in combination with compass and tilt, pressure, current and sound velocity data, is expected to measure the positions of the digital optical modules in the deep sea with an accuracy of about $10 \mathrm{~cm}$. Since data are continuously transmitted to shore and distributed to the local data acquisition network at the shore station, acoustic data are available also for Earth and Sea science users. The KM3NeT APS is also an excellent tool to study the feasibility of an acoustic neutrino detector and a possible correlation between acoustic and optical signals.

The 34th International Cosmic Ray Conference,

30 July- 6 August, 2015

The Hague, The Netherlands

*Speaker. 


\section{Introduction}

KM3NeT is a multi- $\mathrm{km}^{3}$ sized neutrino telescope that will be installed in the Mediterranean Sea [1]. The detection principle is based on the measurement of the Cherenkov light induced by relativistic charged particles emerging from an interaction of a neutrino in the vicinity of the detector. After the first phase of the construction, namely KM3NeT Phase 1, the detector will consists of 24 Detection Units (DUs) deployed off-shore Capo Passero, in Italy, (KM3NeT-IT) and 7 DUs, deployed off-shore Toulon, in France (KM3NeT-FR). Each DU hosts 18 digital optical modules (DOMs), each one equipped with 31 photo-multipliers tubes (PMTs) [2]. Oceanographic properties of the installation site will be monitored by dedicated structures, called Calibration Units (CUs). DUs and CUs will be connected by means of junction boxes (JBs) with an electro-optical seafloor network. During the telescope operation, in order to effectively reconstruct muon tracks, generated by the interaction of cosmic neutrinos with water nuclei, via the optical Cherenkov technique, the coordinates of the optical sensors must be known (relative to a well-defined reference system) with an accuracy of about $10 \mathrm{~cm}$. In the deep sea, DUs are anchored to the sea bed but they are free to move along their vertical expansion under the effect of currents, thus their positions must be determined and monitored. In KM3NeT, thanks to a long baseline (LBL) of acoustic transmitters placed on the seabed in known positions and an array of acoustic receivers rigidly connected to the mechanical structures of the telescope, the optical sensor positions are continuously calculated via triggered emission of acoustic signals. Their repetition rate could be varied from about once per minute (under conditions of low sea currents, few $\mathrm{cm} / \mathrm{s}$, as measured in deep-sea sites) to higher rates in conditions of more intense currents. In the range of frequencies $20 \div 40 \mathrm{kHz}$, acoustic signals can effectively propagate in water: at $2 \mathrm{~km}$ distance the amplitude for a $180 \mathrm{~dB}$ re $1 \mu \mathrm{Pa}$, $32 \mathrm{kHz}$ tone is about $110 \mathrm{~dB}$ re $1 \mu \mathrm{Pa}$ and it can be easily recognised by the acoustic receivers of the telescope.

\section{The Long Base-Line (LBL)}

The LBL of KM3NeT is composed of an array of acoustic transmitters and receivers hosted on the DUs bases and on CUs bases (CBs). Each DU base will host a digital hydrophone, each CB will host an acoustic beacon and a digital hydrophone placed at known distance from the beacon. These "built-in" acoustic devices will be connected to the detector power and data transmission system and they are driven by the master clock of the detector [3]. Since the submarine network of the KM3NeT-IT seabed infrastructure in Capo Passero does not foresee CBs, the two secondary Junction Boxes of the KM3NeT-IT site will be used for positioning purposes as calibration bases. The LBL of the acoustic beacons installed on the CBs is complemented by an array of additional autonomous acoustic beacons, placed outside the footprint of the telescope, improving the resolution of multi-lateration calculation for receivers placed on the DUs at the edge of the telescope field. Autonomous beacons will have a battery pack and will be placed on tripods, for easy positioning and possible re-displacement. During the installation of the first DUs, autonomous beacons must be used to create the LBL field and permit implementation of LBL where only few structures are deployed and connected. Following ANTARES [4] and NEMO [5] experience, the expected accuracy on LBL elements position is about $1 \mathrm{~m}$. The main characteristic of the LBL system of 
$\mathrm{KM} 3 \mathrm{NeT}$ is the time synchronisation with the detector master clock. This allows the implementation of two mandatory characteristics of the system: LBL auto-calibration and the possibility to accurately measure Time of Flight (ToF) of acoustic signals emitted by each beacon and received by the acoustic receivers with the same absolute clock. For this purposes, the time synchronization and calibration with respect to the detector master clock must be very accurate and stable. The expected accuracy in determination of their relative positions after the auto-calibration procedure is of the order of a few $\mathrm{cm}$. Each LBL unit is composed of: an acoustic beacon assembly (piezo ceramic, electronics board, pressure gauge), and an acoustic receiver placed on each $\mathrm{CB}$ at known distance from the beacon. The commercially available piezo-ceramic Free Flooded Rings (FFR) transducers SX30 from Sensor Tech. Ltd [6] have been chosen for KM3NeT positioning purposes. The unlimited depth for operation, the working frequency range $(20 \div 40 \mathrm{kHz})$, the transmitting and receiving voltage responses $(133 \mathrm{~dB}$ re $1 \mu \mathrm{Pa} / \mathrm{Volt} @ 1 \mathrm{~m}$ and $-183 \mathrm{~dB}$ re $1 \mathrm{Volt} / \mu \mathrm{Pa}$, respectively), the input power (300W during a $2 \%$ duty cycle) and low cost, make these transducers a very good option for the proposed application. These emitters were tested at Universitat Politècnica de València (UPV) for calibration checks at 1 bar pressure and at the HIPERBARIC company in Burgos (Spain) up to 400 bar, in a high pressure machine model Hiperbaric 420 [7]. The acoustic beacon assembly for KM3NeT, shown in Fig.1, is produced by the Mediterráneo Señales Marìtimas company [8].

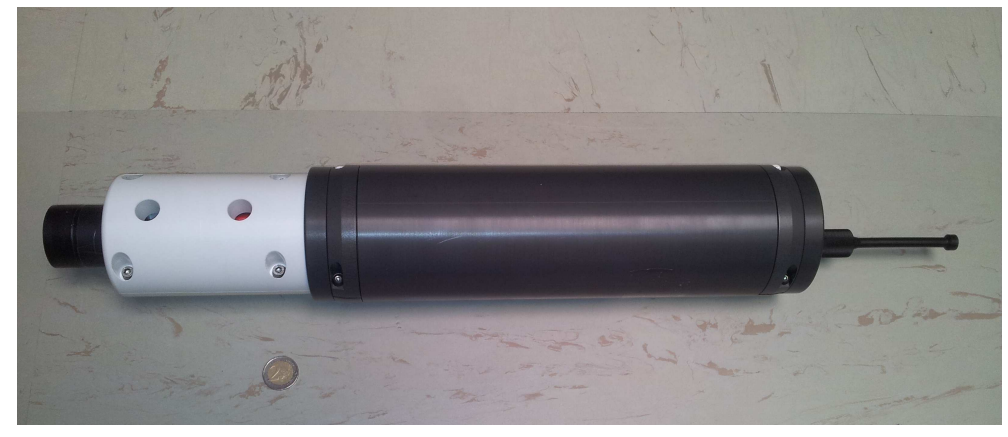

Figure 1: Acoustic beacon assembly used for the KM3NeT LBL.

LBL beacons can be reconfigured via a dedicated RS232 connection with a Central Logic Board (CLB) installed on the CB electronics, in order to allow reconfiguration of acoustic emission signal parameters (amplitude, waveform, timing) for "in situ" optimization of the signal detection. The signal emission trigger is received from the CLB on a $\mathrm{CB}$, synchronized with the detector master clock. The positions of acoustic beacons and receivers on the DU bases must be geo-referenced during the deployment operation using a USBL (Ultra-Short BaseLine) system available on board the ship that performs the deployment. In KM3NeT-IT this system relies on a self-consistent set-up made of a transmitting/receiving USBL transponder array installed aboard the ship and on several transponders, installed on submarine vessels (e.g. ROVs) or submersible structures. Following the KM3NeT-IT experience, the USBL positioning procedure has accuracy close to $1 \mathrm{~m}$. For KM3NeT-FR the positioning of the LBL elements will be performed thanks to a custom version of the RAMSES positioning system [9]. In this system, the installation of an additional set of few USBL autonomous transponders, moored on tripods at the sides of the installation 
field is foreseen. This will permit, with minimal costs to set a reference and quickly re-calibrate the USBL system installed on the deployment ship during sea operation and facilitate the ROV navigation inside the telescope volume. The detection of USBL transponder signals by the LBL receivers will also permit cross-calibration of the USBL and LBL.

\section{Receiving acoustic array}

Digital Acoustic Receivers (DARs) are installed in each DOM of the Detection Units (DUs), on DU bases and on CBs. ToF measurements between LBL beacons and acoustic receivers on the DOMs will be used to reconstruct the DOM's positions via multi-lateration (thus, a minimum number of 4 beacons must be active and detected by each receiver). The expected accuracy in the determination of the DOM's position with respect to the LBL reference system is about 10 $\mathrm{cm}$. In each DAR the analogue signals are pre-amplified and digitized by a dedicated electronic board placed in the proximity of the receiver. The clock signal of each DAR is recovered from the detector master clock and provided by the CLB, an FPGA-based board dedicated to the packaging of both the optical sensors signals and those from the calibration instrumentation into an Ethernet protocol for transmission over the optical network to shore [3]. The master clock frequency derived from the CLB is $25 \mathrm{MHz}$ and the data sampling frequency is about $195.3 \mathrm{kHz}$. The used Sigma-Delta ADCs permit amplitude quantization of 24 bit and possibility to digitize the signal with 2 different gains (high and low) using two independent transmission channels (stereo stream). Data from each DAR are sent and treated by the CLB, which time-stamps the received data and can select, via proper command from shore: 1) to propagate one or both data streams received from the DAR, 2) to reduce the number of amplitude bits to be sent to shore These options allow flexibility of the system, aimed at adjusting the dynamic range of the DAR for low and high amplitude signals and permit also the reduction of the data flow to shore. The use of AES/EBU (Audio Engineering Society/ European Broadcasting Union) protocol simplifies interface with CLB and permit to use standard audio libraries. The absolute GPS time is stamped in the data stream by the CLB, tagging unambiguously the acoustic signal detection time. The CLB sends continuously the received acoustic data stream to shore, embedded in the main DOM data-stream. Since all DOMs of the detector are synchronous and phased with respect to the GPS time signal transmitted from shore, the whole acoustic receivers array is synchronized to the GPS master clock. Two kinds of technologies for acoustic sensors are available and will be installed aboard the DUs: an "internal" digital piezoelectric-sensor, installed inside the DOM and a digital "external" hydrophone, installed in each DU base.

\section{The internal piezo sensor}

The "internal" piezo-electric digital acoustic receiver is glued from the inside to the glass sphere of each KM3NeT DOM, as shown in Fig.2. Within the sphere, pressure is slightly below atmospheric value, cooling is achieved by the ambient water at a temperature of about $13^{\circ} \mathrm{C}$. The sensor is equipped with a cylindrical piezo ceramics with a diameter of $18 \mathrm{~mm}$ and a height of 11.5 $\mathrm{mm}$. 


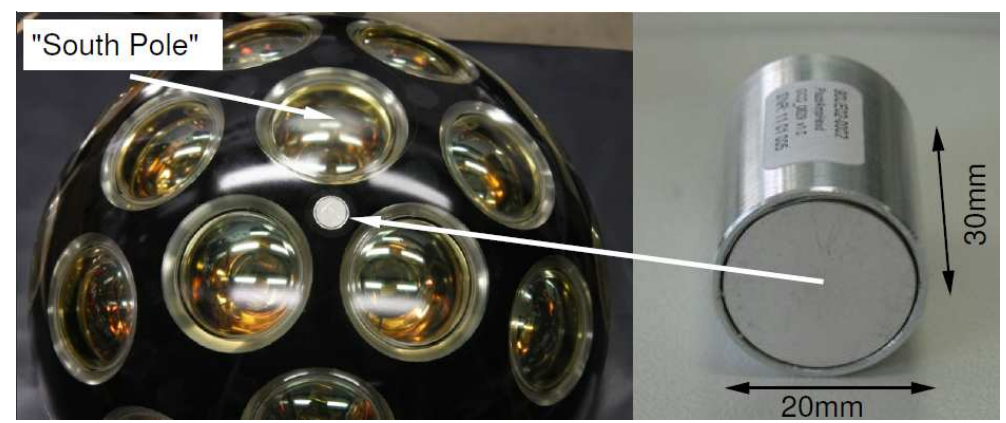

Figure 2: Left: Piezo-electric digital acoustic receiver integrated in a KM3NeT DOM. Right: Piezo-electric digital acoustic receiver in more detail.

The design sensitivity of the sensors is $-160 \pm 6 \mathrm{~dB}$ re $1 \mathrm{~V} / \mathrm{Pa}$ at $50 \mathrm{kHz}$ with a $\pm 3 \mathrm{~dB}$ variation (long time average) in the range $10 \div 70 \mathrm{kHz}$. However, these specifications hold when the sensor is glued to the inside of the glass sphere of a $\mathrm{KM} 3 \mathrm{NeT}$ optical module and its response to pressure signals depends very strongly on the operation environment of the sensor. The performance of the digital piezo sensor has been investigated in the laboratory. In particular, as it is known that the operation of the PMTs within a DOM produces electronic noise in the electronics of the piezo sensor, it had to be verified that this interference does not compromise the potential for position reconstruction of the sensor. In Fig. 3 the power spectral density of the electronic noise of a piezoelectric sensor inside a DOM in cases of PMTs turned on and PMTs turned off is reported. With the PMTs off, the distributions show a very flat behaviour in the range $10 \mathrm{kHz}$ to $80 \mathrm{kHz}$. Beyond that frequency the anti-alias filter sets in. Below $5 \mathrm{kHz} 1 / \mathrm{f}$ noise leads to a strong rise of the noise. At about $200 \mathrm{~Hz}$ the effect of a high pass filter sets in (not visible in the figure), which is intended to prevent saturation of the sensor due to strong low frequency ambient sea-noise. The peak at $0 \mathrm{~Hz}$ corresponds to a DC offset of about 3-4 $\mathrm{mV}$ at the analogue input of the ADC which is not significant relative to the full input range of $4 \mathrm{~V}$ and can be easily removed in the analysis. The operation of the PMTs produces spikes at distinct frequencies in the spectrum. However, in the frequency range from 20 to $40 \mathrm{~Hz}$, which is relevant for position reconstruction, the effect is minimal. The RMS of the noise was measured to be about $2000 \mathrm{LSB}\left(1 \mathrm{LSB}=4 \mathrm{~V} / 2^{24}\right)$ for the full range and about $600 \mathrm{LSB}$ for the frequency range above $5 \mathrm{kHz}$.

\section{The external digital hydrophone}

External hydrophones will be hosted on the base of the DUs and on CBs, where no DOMs are foreseen. These hydrophones will be used to calibrate the LBL measuring the relative distances among LBL elements and - when autonomous beacons are used- to calculate the times of emission (ToEs) of the autonomous beacons. The selected hydrophone, shown in Fig.4 is the DG0330 manufactured by Colmar s.r.1.[11].

It consists of a spherical piezo-ceramic element, read-out by an analogue board splitting the signal in two lines with different gains ( $+46 \mathrm{~dB}$ and $+26 \mathrm{~dB}$ respectively). The double gain feature has been implemented to avoid signal saturation when the hydrophone is placed close (about 1 


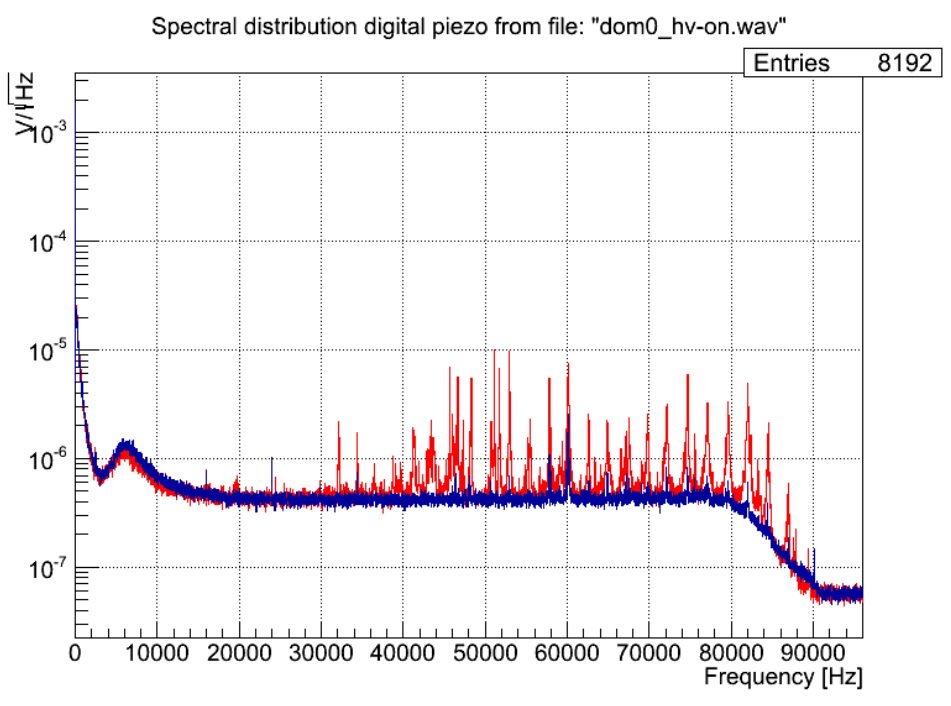

Figure 3: Comparison of piezo power spectral density as measured within a DOM in the laboratory. The blue curve shows the distribution for PMTs power off, the red curve for PMTs power on.

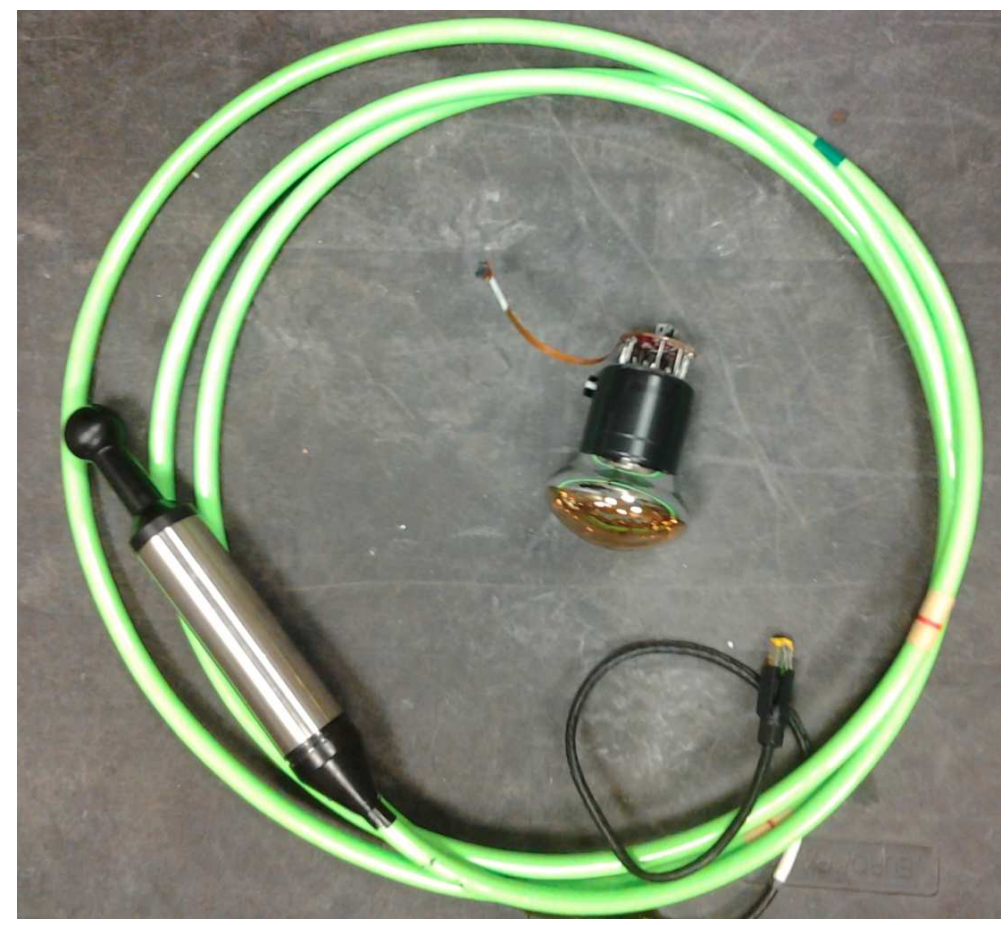

Figure 4: The digital Colmar hydrophone used as external hydrophone in KM3NeT.

meter) to the beacon -as in the case of Calibration Bases- and use the high gain channel to analyze data received from far (up to a few $\mathrm{km}$ ) beacons and to study fainter acoustic signals (bio-acoustics, environmental noise, acoustic neutrino detection). The two streams are sampled by a stereo 24 bit 
ADC (CS-4270) and converted into AES/EBU protocol using a DIT (Digital Interface Transmitter). Two hydrophone versions were designed, without and with an analogue signal high-pass filtering stage. The filter frequency is $700 \mathrm{~Hz}$, to reject the low frequency ambient sea-noise (which follows a $1 / \mathrm{f}$ shape, and flattens at about $5 \mathrm{kHz}$ ) thus improve Signal to Noise ratio in the detection of beacon pulses (20-40 kHz). The not-filtered version can be also used for acoustic noise monitoring and marine bio-acoustics. The typical noise spectrum of a filtered DG0330 hydrophone is reported in Fig.5.

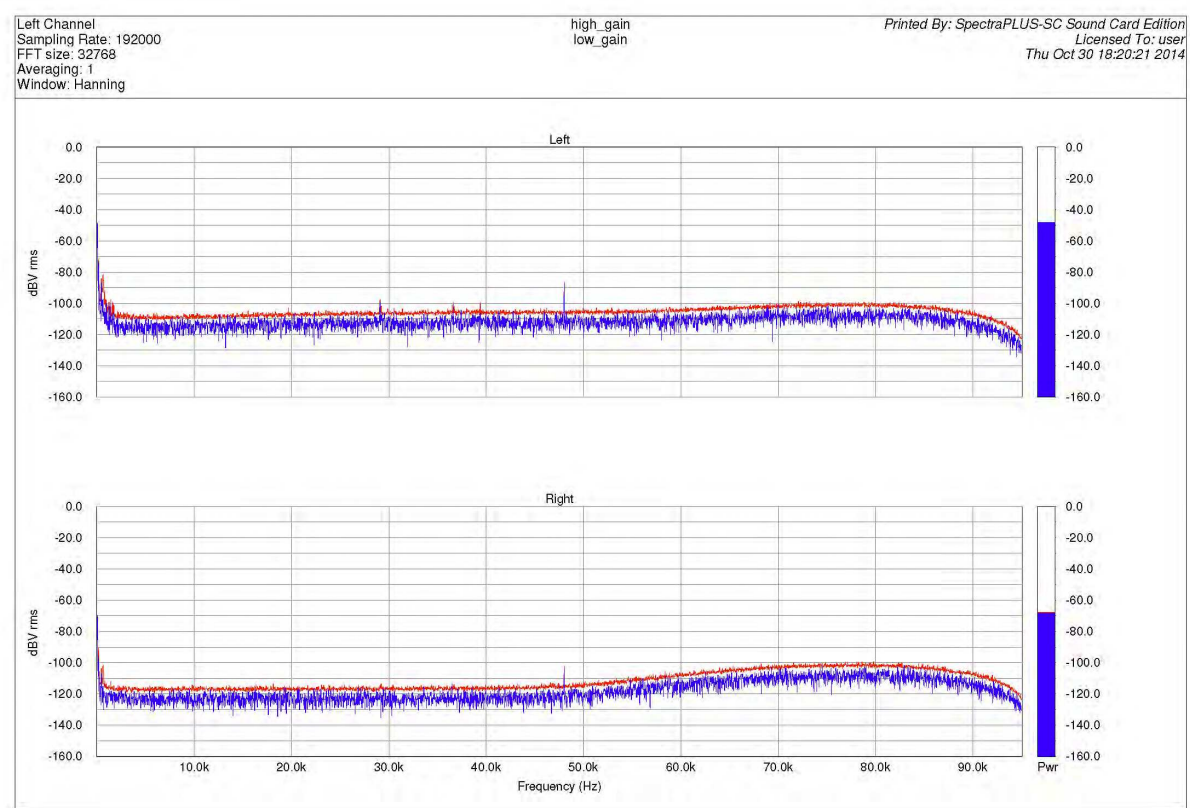

Figure 5: Typical noise spectrum and total noise of a filtered DG0330 hydrophone, measured during hydrophone acceptance test at INFN-LNS. Upper plot high-gain channel; lower plot low-gain channels.

For the first DUs to be deployed, the version of the DG0330 with high-pass filter will be used. The time latency of the hydrophone electronics (including $4.5 \mathrm{~m}$ cable) has been measured using a dedicated setup and its value is $50.65 \pm 0.02 \mu$ s for the low gain channel and $50.71 \pm 0.02 \mu$ s for the high gain channel.

\section{DOM positioning procedure}

The Acoustic Positioning data analysis is performed on-shore by a farm of PCs. These PCs receive, on a intranet, data from the DARs, parsed from the main data stream. Data can be dispatched on shore either in native AES/EBU format (that permit easy interfacing with standard acoustic libraries and software) or in any other format that allows conservation of acoustic information (24 bits/channel, sampling frequency $195 \mathrm{kHz}$, absolute time header). The ToA of the acoustic signals detected by DARs are measured, analysing the DAR raw data flow using software algorithms based on matched filters. This step requires that the filtering algorithms have, as input, the characteristics 
of the signal emitted by each single active beacon, such as frequency and emission pattern. The waveform of the signal and the absolute time of emission (ToE) of each acoustic beacon to be emitted are set during run initialization and configuration, and are stored in the data base. Once a LBL-beacon pulse is identified, the code associates to it the detection GPS time (ToA). The distance between each beacon and each DAR will be then calculated taking into account the sound velocity profile along the water column. Knowing the location of the LBL beacons on the seabed, each DAR positions is recovered using algorithms based on spherical multi-lateration . At last, the calculated DARs positions are stored in the detector database and visualized by a dedicated Graphical User Interface.

\section{Conclusions}

The expected accuracy of the KM3NeT APS for DOM positioning is estimated to be of about $10 \mathrm{~cm}$, allowing a precise reconstruction of Cherenkov neutrino-induced events. Since all acoustic data are continuously transmitted to shore, DAR data could be shared with the Earth and Sea Science Community for several research items: marine monitoring, biology, geophysics, oceanography, etc. In addition, thanks to the synchronization of each DAR with the Master Clock of the telescope, the KM3NeT APS represents a good tool to study the feasibility of an acoustic neutrino detector and the possible correlation between acoustic and optical signals induced by neutrino events [12].

\section{References}

[1] KM3NeT website, http://www.km3net.org

[2] R. Bruijn and D. van Eijk, The KM3NeT Multi-PMT Digital Optical Module, these conference proceedings.

[3] KM3NeT Technical Design Report, www.km3net.org/TDR/TDRKM3NeT.pdf

[4] S. Adrián-Martínez, et al., Journal of Instrumentation 7 T08002 (2012) 1-20

[5] S. Viola et al., Nuclear Instruments and Methods in Physics Research A 725 (2013) 207-210

[6] Sensor Technology Ltd website, http://www.sensortech.ca

[7] Hiperbaric website, http://www.hiperbaric.com/en

[8] Mediterráneo Señales Marìtimas website, http://www.mesemar.com

[9] iXBlue-Ramses webpage, http://www.ixblue.com/products/ramses

[10] Ferroperm website, http://www.ferroperm-piezo.com

[11] Colmar s.r.l website, http://www.colmaritalia.it

[12] L. Lahmann, arXiv:1304.0697v1 [astro-ph.IM] 2013, http://arxiv.org/pdf/1304.0697.pdf 